\title{
Chronic Pulmonary Aspergillosis - Case Series and Review of Indian Literature
}

\author{
Vikas Marwah, Robin Choudhary, Deepu K. Peter, Gaurav Bhati, Kunal Kumar, Akhil R, Shafin Babu \\ Department of Pulmonary, Critical Care and Sleep Medicine, Army Institute of Cardiothoracic Sciences, Pune, Maharashtra, India
}

\section{Abstract}

Aspergillus species, especially Aspergillus fumigatus, can cause varied pulmonary manifestations depending on the host immune status and duration of infection. Chronic pulmonary aspergillosis (CPA) is an under-reported entity, and its diagnosis remains a diagnostic challenge for clinicians. CPA can have varied presentations including chronic cavitary pulmonary aspergillosis (CCPA), subacute invasive pulmonary aspergillosis, and chronic fibrosing pulmonary aspergillosis. Underlying lung diseases such as chronic obstructive pulmonary disease and sequelae of pulmonary tuberculosis predisposes these individuals to CPA. These cases can present as chronic cough with hemoptysis even requiring emergency bronchial artery embolization. Here, we present a series of four CCPA cases who were diagnosed on the basis of clinico-radiological features and the Aspergillus-specific IgG antibody levels. A review of case series published in India is also presented.

Keywords: Aspergillus-specific IgG, chronic pulmonary aspergillosis, voriconazole

\section{INTRODUCTION}

Chronic pulmonary aspergillosis (CPA) includes a spectrum of manifestations such as chronic cavitary pulmonary aspergillosis (CCPA), subacute invasive pulmonary aspergillosis (IPA), and chronic fibrosing pulmonary aspergillosis (CFPA) ${ }^{[1,2]}$ A disease duration of more than 3 months distinguishes CPA from acute and subacute pulmonary aspergillosis. These diseases are generally seen in individuals with prior structural lung diseases including sequelae of pulmonary tuberculosis, allergic bronchopulmonary aspergillosis, sarcoidosis, lung cancer, emphysema, and chronic obstructive pulmonary disease (COPD). ${ }^{[3,4]}$ However, the disease entity of subacute IPA is also seen in individuals with variable degree of immunosuppression such as diabetes, human immunodeficiency virus (HIV), and alcoholism..$^{[5,6]}$ Herein, we present a case series of four cases of CCPA and a review of Indian literature.

\section{Case Reports}

\section{Case 1}

A 56-year-old man with morbid obesity was admitted to our hospital with multiple episodes of hemoptysis of about $50 \mathrm{ml}$ each and shortness of breath for the last 4 years. He was a known case of Type 2 diabetes mellitus and primary

\begin{tabular}{|l|l|}
\hline \multicolumn{3}{|c|}{ Access this article online } \\
\hline Quick Response Code: & Website: \\
\hline & www.ijrc.in \\
\hline
\end{tabular}

hypertension. His diabetes mellitus was poorly controlled and he was on oral hypoglycemic drugs. His vital parameters were within normal limits. His general examination revealed bilateral pitting pedal edema. On evaluation, his laboratory data showed normal hemogram (hemoglobin $15.9 \mathrm{~g} / \mathrm{dl}$, total leukocyte count 7500/cumm, differential count - neutrophils $65 \%$, lymphocytes $25 \%$, platelets 2.5 lakhs/cumm), blood urea $40 \mathrm{mg} / \mathrm{dl}$, and serum creatinine $1.6 \mathrm{mg} / \mathrm{dl}$. His liver function tests and coagulation profile were normal. His electrocardiogram showed left ventricular hypertrophy. Echocardiography confirmed the same along with Grade I diastolic dysfunction. Chest radiograph revealed reticulonodular opacities in the right middle and lower zones. He underwent a computed tomogram of the chest which elucidated subsegmental collapse consolidation in the superior lingular segment of the left upper lobe and collapse consolidation in the right middle

Address for correspondence: Dr. Robin Choudhary, Department of Pulmonary, Critical Care and Sleep Medicine, Army Institute of Cardiothoracic Sciences, Pune - 411 040, Maharashtra, India. E-mail: robinch19@gmail.com

This is an open access journal, and articles are distributed under the terms of the Creative Commons Attribution-NonCommercial-ShareAlike 4.0 License, which allows others to remix, tweak, and build upon the work non-commercially, as long as appropriate credit is given and the new creations are licensed under the identical terms.

For reprints contact: WKHLRPMedknow_reprints@wolterskluwer.com

How to cite this article: Marwah V, Choudhary R, Peter DK, Bhati G, Kumar K, Akhil R, et al. Chronic pulmonary aspergillosis - Case series and review of Indian literature. Indian J Respir Care 2021;10:230-5.

Received: 11-09-2020 Revised: 04-02-2021

Accepted: 05-04-2021 Published: 14-06-2021 
lobe [Figure 1a]. During the course of hospitalization, he had an episode of massive hemoptysis. Emergency bronchoscopic cold saline and adrenaline irrigation was performed which helped in the temporary control of hemoptysis. However, in view of persisting symptoms, his bronchoscopy was repeated, and his bronchial wash fluid and postbronchoscopic sputum was sent for microscopic studies and appropriate cultures. The microscopic studies revealed narrow septate fungal hyphae with acute angle branching, presumptively, Aspergillus species, and no malignant cells were seen. The Aspergillus-specific IgG was raised $(45 \mathrm{mgA} / \mathrm{l})$ in this patient.

He was commenced on antifungal agents (oral voriconazole with dosing of $400 \mathrm{mg}$ twice daily for $24 \mathrm{~h}$ and $200 \mathrm{mg}$ twice daily for 6 weeks). He was also administered injectable antifungals during the initial acute period. The patient showed significant improvement clinically and had no further episodes of hemoptysis. Repeat computed tomogram of the chest, after 6 weeks of antifungal treatment, showed that resolution of collapse consolidation of the right middle and inferior lingular segment of the left upper lobe and ground-glass attenuation in the superior lingular segment had also resolved [Figure 1b].

\section{Case 2}

An 83-year-old man, a reformed smoker, reported to our hospital with a history of recurrent episodes of hemoptysis of about $50 \mathrm{ml}$ each for 1 month. On examination, he was afebrile with a respiratory rate of $18 / \mathrm{min}$ and was maintaining a saturation of $98 \%$ at room air. His complete blood counts and renal and liver function tests were within normal limits. His chest radiograph showed bronchiectatic changes in the right middle and lower lobes and in the lingula [Figure 2]. He was initially managed with injectable antibiotics and supportive management. However, he had an episode of life-threatening hemoptysis, for which he underwent emergency bronchial artery embolization (BAE). He continued to have streaky hemoptysis post-BAE. He was further evaluated for CPA, and his serum Aspergillus-specific antibody ( $\mathrm{IgG})$ was raised - $42.90 \mathrm{mgA} / 1$ (normal range $<27 \mathrm{mgA} / \mathrm{l}$ ). He was diagnosed as a case of CPA and was commenced on oral antifungal agents (voriconazole with dosing of $400 \mathrm{mg}$ twice

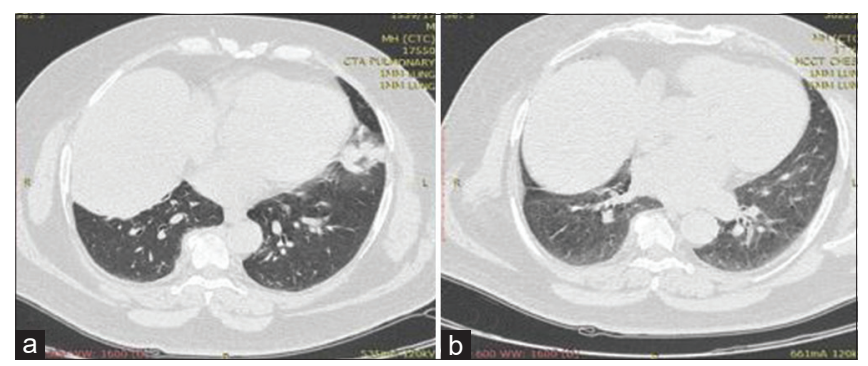

Figure 1: (a) High-resolution computed tomography of the chest on December 22, 2018, showing subsegmental collapse consolidation in the superior lingular segment of the left upper lobe. (b) High-resolution computed tomography of the chest on February 5, 2018, revealing good resolution of the consolidation of lingular segment of the left upper lobe daily for $24 \mathrm{~h}$ and $200 \mathrm{mg}$ twice daily for 6 weeks), to which he showed significant clinico-radiological response.

\section{Case 3}

A 24-year-old male with no known comorbidities presented with a history of fever and cough with mucoid expectoration of 3 weeks' duration. He gave a history of similar complaints 10 years and 2 years ago, for which he was treated with empirical antituberculous drugs for 6 months. On evaluation, he was afebrile and his vital parameters were within normal limits. His complete blood counts and renal and liver function tests were essentially normal. His chest skiagram showed fibronodular opacities in the right upper and middle zone [Figure 3a]. He underwent computed tomography of the chest which showed cylindrical bronchiectasis in the bilateral lower lobes (right $>$ left) with air space consolidation with breakdown and thick-walled multiple cavities in the apical segment left lower lobe [Figure $3 \mathrm{~b}$ and $\mathrm{c}$ ]. He was extensively evaluated and underwent videobronchoscopy. The bronchoalveolar lavage (BAL) sample sent for acid-fast bacilli/mycobacteria growth indicator tube was negative for Mycobacterium tuberculosis and malignancy. Although the fungal stain of BAL fluid was negative, he was suspected to have chronic fungal infection and his serum Aspergillus-specific IgG was raised $(>43.00 \mathrm{mgA} / \mathrm{l})$. He was diagnosed as CPA and was started on oral voriconazole. He showed significant clinical response after 6 weeks of antifungal treatment. He is presently asymptomatic.

\section{Case 4}

A 65-year-old male reported with a history of cough with blackish sputum and weight loss for 1-month duration. He had similar complaints in the past 4 years, for which he was managed conservatively. On evaluation, he was found to have anemia $(\mathrm{Hb}-9.3 \mathrm{mg} / \mathrm{dl})$. His chest skiagram showed two cavitary lesions in the right upper and middle zone with surrounding consolidation. His computed tomography of the chest showed thick-walled cavitary lesion $(4.7 \mathrm{~cm} \times 4.8 \mathrm{~cm} \times 8.2 \mathrm{~cm})$, small multiple cavitary

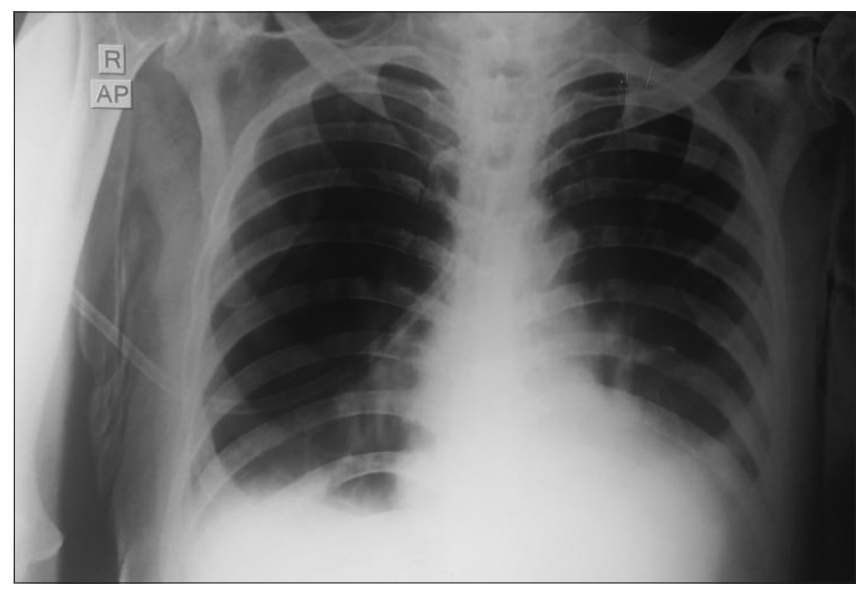

Figure 2: Chest radiograph showing bronchiectatic changes in the right middle and lower lobes. 
Marwah, et al.: Chronic pulmonary aspergillosis - Case series

nodules in the bilateral upper lobe, and multiple nodules in the right middle lobe and left lower lobe. He was initially started on empirical antituberculous drugs, but there was no improvement even after 4 months of antitubercular therapy. He underwent fiber-optic bronchoscopy and histopathological examination of his endobronchial biopsy showed areas of inflammatory infiltrates and necrosis with few fungal colonies, thin septate hyphae with acute angle branching, suggestive of aspergillosis. The hyphae showed frequent septations and regular branching and were seen at hematoxylin and eosin stain and highlighted by Gomori methamine silver stain [Figure $4 \mathrm{a}$ and $\mathrm{b}]$. His Aspergillus-specific IgG was also raised ( $>44.5 \mathrm{mgA} / \mathrm{l})$. He was managed as a case of CCPA and was commenced on liposomal injection amphotericin-B (dose - $5 \mathrm{mg} / \mathrm{kg} /$ day). However, he developed infusion reaction with injection amphotericin, and on evaluation, his electrocardiography showed ST-T changes, his Trop-T was positive, and two-dimensional echocardiography showed apical dysfunction and ballooning. He was diagnosed as a case of acute coronary syndrome with cardiogenic shock (Takotsubo syndrome) and was managed with continuous positive airway pressure and dobutamine infusion. Injection amphotericin-B was stopped and was switched over to injection caspofungin. He also developed coffee-colored aspirate in Ryle's tube, a drop in his hemoglobin levels, and signs of multiorgan failure. He was administered packed red cells and fresh frozen plasma. He was later intubated due to persistent tachypnea, rising arterial carbon dioxide and respiratory fatigue, and mechanically ventilated. He developed critical care myoneuropathy and underwent elective tracheostomy in view of prolonged ventilation. He was continued on injection caspofungin, oral voriconazole, and mechanical ventilatory support. He showed significant response to these and was later discharged with tracheostomy tube with speaking valve and oral voriconazole. He is presently asymptomatic, and his tracheostomy has been decannulated.

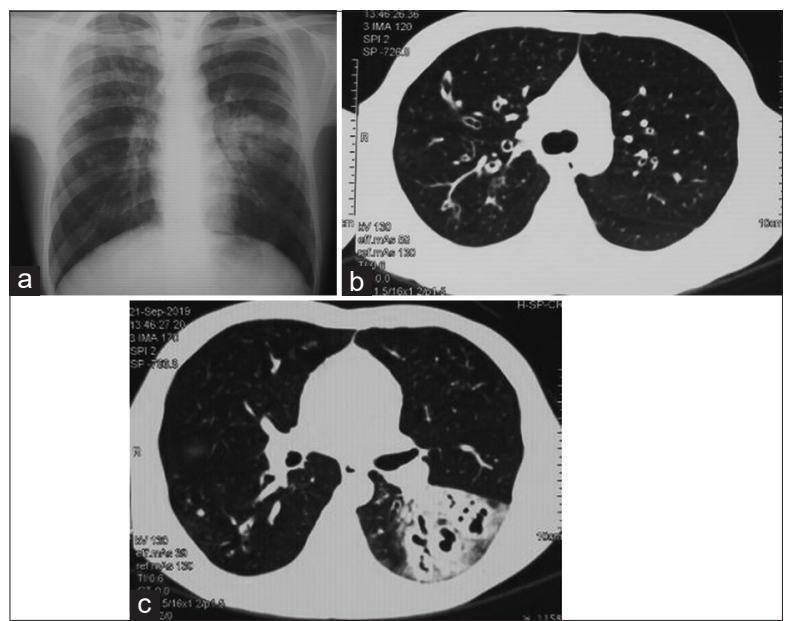

Figure 3: (a) Chest skiagram showing fibronodular opacities in the right upper and middle zone. (b) Computed tomography of the chest showing cylindrical bronchiectasis bilateral lower lobe. (c) Computed tomography of the chest showing air space consolidation with breakdown and thick-walled cavities in the apical segment left lower lobe
The characteristics of patients and outcomes are summarized in Table 1.

\section{Discussion}

Aspergillus species is ubiquitous in nature and has various manifestations depending on the duration of infection and immune status of the host. CPA is almost always caused by Aspergillus fumigatus, although Aspergillus niger and Aspergillus flavus have also been identified in certain patients. ${ }^{[7,8]}$ CPA was first recognized as a fatal condition in 1842 in Edinburgh, United Kingdom. Patients with underlying pulmonary diseases such as COPD, posttuberculosis sequelae, cystic fibrosis, and bronchiectasis are more predisposed to develop CPA. Previously treated tuberculosis is one of the most common predisposing risk factors, worldwide..$^{[9,10]}$

The affected individuals are generally middle-aged males who present with constitutional symptoms (weight loss, malaise, sweats, and anorexia), chronic productive cough, dyspnea, and occasional hemoptysis. Individuals with immunosuppression such as HIV or those on steroids can have rapid progression of disease ${ }^{[10]}$ All our patients were male and were more than 55 years of age except one, and they had underlying existing lung disorders such as COPD or posttuberculous sequelae.

Chronic forms of Aspergillus infection in the lung include chronic necrotizing pulmonary aspergillosis, CCPA, aspergilloma, and CFPA. The terminology of CPA has been developed to describe a spectrum of disease entities that have considerable overlap and variation in severity. ${ }^{[10,11]}$ The most common form of CPA is CCPA, and it can lead to CFPA if not adequately treated. Aspergillus nodule and single aspergilloma constitute less common form of CPA.

On imaging, CPA is characterized by cavities, consolidation, and paracavitary opacities. ${ }^{[9]}$ Single aspergilloma generally constitutes of a single pulmonary cavity with intracavitary fungal ball. CCPA is described radiologically with one or

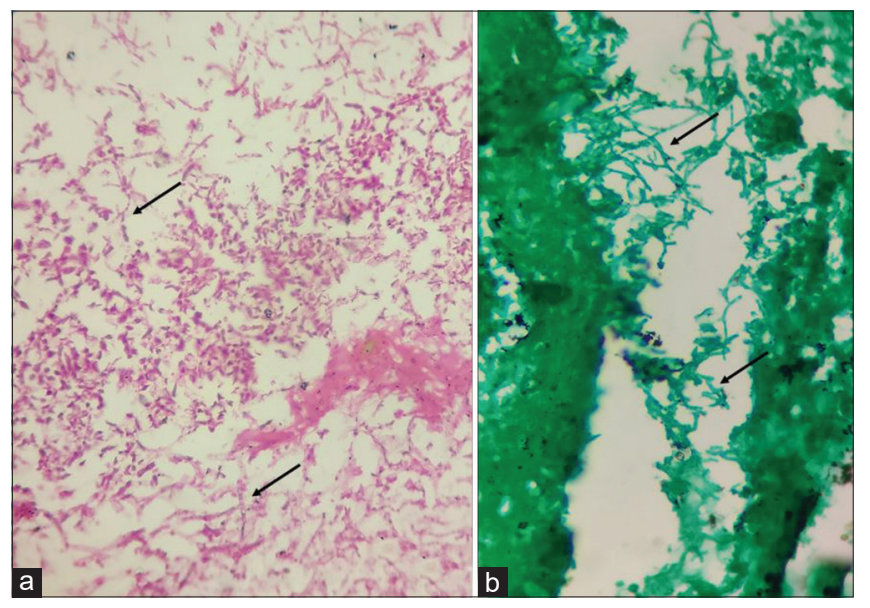

Figure 4: (a) Histopathological slide $(H$ and $E$ stain, $\times 400)$ showing thin septate hyphae with regular, acute angle branching (marked by arrows). (b) Histopathological slide (GMS stain, $\times 400$ ) highlighting the fungal hyphae (marked by arrows) 
Marwah, et al.: Chronic pulmonary aspergillosis - Case series

\begin{tabular}{|c|c|c|c|c|c|}
\hline Age (years) & Sex & Presenting complaints & CT findings & $\begin{array}{l}\text { Serum IgG }(\mathrm{mgA} / \mathrm{l}), \\
\text { (normal-<27) }\end{array}$ & Treatment \\
\hline 56 & Male & Hemoptysis, dyspnea & $\begin{array}{l}\text { Subsegmental collapse-consolidation in the superior } \\
\text { segment of left upper lobe and right middle lobe }\end{array}$ & 45 & Voriconazole \\
\hline 83 & Male & Hemoptysis & $\begin{array}{l}\text { Bronchiectatic changes in right middle lobe, lingual, and } \\
\text { lower lobes }\end{array}$ & 42.90 & Voriconazole \\
\hline 24 & Male & Cough with expectoration & $\begin{array}{l}\text { Bronchiectasis bilateral lower lobes, air space consolidation } \\
\text { with breakdown and thick-walled cavities in the apical } \\
\text { segment left lower lobe }\end{array}$ & 43.00 & Voriconazole \\
\hline 65 & Male & $\begin{array}{l}\text { Cough with } \\
\text { expectoration, weight loss }\end{array}$ & $\begin{array}{l}\text { Thick-walled cavitary lesion with cavitary nodules in the } \\
\text { bilateral upper lobes, right middle lobe, and left lower lobe }\end{array}$ & $>44.5$ & $\begin{array}{l}\text { Caspofungin, } \\
\text { voriconazole }\end{array}$ \\
\hline
\end{tabular}

CT: Computed tomography

more pulmonary cavities, which can be thin/thick walled with intraluminal aspergilloma or irregular intraluminal content which shows radiological progression over at least 3 months. CFPA generally shows severe fibrotic destruction of at least two lobes of the lung, which can appear as large cavities with surrounding fibrosis. ${ }^{[9-11]}$

The presence of $A$. fumigatus in the sputum sample is not significant due to ubiquitous nature of the fungus, but the presence of the same in the bronchoscopic fluid sample is more conclusive, especially culture growth of Aspergillus. ${ }^{[12]}$ Detection of Aspergillus antibodies is indispensable for diagnosis off CPA since it differentiates between infection and colonization and has positive predictive value of $100 \%$. Aspergillus-specific antibodies have a sensitivity of $80 \%-90 \%$. Other serological tests include detection of serum galactomannan (sensitivity $77 \%$ ) and $\beta$-D-glucan (sensitivity 20\%), and newer diagnostics include Aspergillus-specific lateral flow device and serum galactoaminogalactan. The newer method of detection also includes real-time polymerase chain reaction which can detect even small amount of fungal DNA and can also be used to detect the resistance against antifungals such as azoles. All the patients in our study had raised Aspergillus-specific antibody $(\operatorname{IgG}) .^{[10,12,13]}$

The mainstay of treatment of CPA is antifungal (triazoles) agents. Oral itraconazole/voriconazole can be given and show good response. CCPA generally show slow response and duration has to be 4-6 months for optimum response. Injectable antifungals such as amphotericin are indicated in progressive disease and where there is intolerance or resistance to oral treatment. Instillation of antifungals in the aspergilloma cavity is indicated for those cases where surgery is not an option to control recurrent hemoptysis. The agents include amphotericin-B, azole, sodium iodide, and nystatin and are generally delivered via a percutaneous catheter or needle under bronchoscopic cover. However, the response to this mode of therapy has been very variable. Surgical resection is a definitive treatment option and should be considered in all patients with severe hemoptysis and cases which are refractory to medical management. BAE is a lifesaving procedure and can also be used as a bridging modality before definitive surgery. The spectrum of surgical modalities includes lobectomy, segmental resection, pneumonectomy or thoracoplasty with simultaneous cavernostomy, and muscle transposition flap. One of our patients required injectable amphotericin for initiation period, while others were managed with oral voriconazole and they showed significant response. ${ }^{[10-12]}$

Moderate-to-severe hemoptysis warrants urgent BAE as a lifesaving measure. It also serves as temporary measure before surgery or definitive treatment. It is successful in $50 \%-90 \%$ of cases. Two of our cases required emergency BAE, and it was successful in both the cases.

\section{Review of Indian literature}

We also did a review of Indian literature and the case series of CPA published in India were included; however, single case reports and editorials were not included. A total of six case series were included in the review [Table 2]. One of the largest studies conducted in Northern India was by Sehgal et al., which included 269 patients with CPA. The median age was 44.3 years with male gender constituting $53.5 \%$. Most common symptoms were cough $(87.4 \%)$, and pulmonary tuberculosis was the most common underlying pulmonary disease in the study population (85.5\%). Aspergillus-specific antibody ( $\operatorname{IgG}>27 \mathrm{mgA} / \mathrm{l})$ was raised in $94.4 \%$ of the study group, and the most common variant of CPA was found to be CCPA (79.9\%). ${ }^{[14]}$ In another study by the same author, 137 patients of CPA (diagnosis was based on clinical, radiological, and serological profile) and confirmed that Aspergillus-specific antibody (IgG) levels of more than $27 \mathrm{mgA} / 1$ had good sensitivity and excellent specificity in diagnosing CPA. In this study, they had also included culture of respiratory sample (sputum and BAL) and other immunological evidence in the form of serum or BAL galactomannan and Aspergillus precipitins. However, the composite sensitivity and specificity of Aspergillus-specific antibody $(\operatorname{IgG})$ were higher than other variables. ${ }^{[15]}$ In another observational and retrospective case series of 22 patients by Chawla et al., the clinical and microbiological profile of all the culture-positive cases of aspergillosis were studied. They concluded that Aspergillus species (especially A. fumigatus) is the most common organism causing chronic pulmonary infection. ${ }^{[16]}$ 
Marwah, et al.: Chronic pulmonary aspergillosis - Case series

\begin{tabular}{|c|c|c|c|c|c|c|}
\hline $\begin{array}{l}\text { Details of } \\
\text { author }\end{array}$ & $\begin{array}{c}\text { Study } \\
\text { population }\end{array}$ & $\begin{array}{c}\text { Median } \\
\text { age (years) }\end{array}$ & $\begin{array}{l}\text { Male: } \\
\text { female }\end{array}$ & Clinical features & Underlying lung disease & $\begin{array}{c}\text { Year of } \\
\text { publication }\end{array}$ \\
\hline Sehgal et al. & 269 & 44.3 & $1.15: 1$ & $\begin{array}{l}\text { Chronic cough, weight } \\
\text { loss, dyspnea }\end{array}$ & Pulm TB (85.5\%) & 2018 \\
\hline Sehgal et al. & 137 & 43.6 & $1: 1$ & $\begin{array}{l}\text { Chronic cough, } \\
\text { hemoptysis }\end{array}$ & Pulm TB (88\%) & 2018 \\
\hline Chawla et al. & 22 & 52.5 & $1.2: 1$ & Chronic cough, dyspnea & $\begin{array}{l}\text { Bronchial asthma, chronic } \\
\text { steroid use }\end{array}$ & 2013 \\
\hline Agarwal et al. & 31 & 37 & $1.3: 1$ & $\begin{array}{l}\text { Cough, dyspnea, } \\
\text { hemoptysis, weight loss }\end{array}$ & Pulm TB $(90 \%)$ & 2013 \\
\hline Kurhade et al. & 20 & Not specified & Not specified & Not specified & Pulm TB, lung abscess & 2002 \\
\hline Shahid et al. & 88 & 49.9 & $5: 1$ & Cough, fever, dyspnea & $\begin{array}{l}\text { Bronchogenic carcinoma, } \\
\text { pulm TB, bronchial asthma }\end{array}$ & 2001 \\
\hline
\end{tabular}

TB: Tuberculosis

In a prospective randomized control trial conducted by Agarwal et al., they confirmed the efficacy of itraconazole in the treatment of patients of CCPA. They conducted the trial on 31 patients after diagnosis of CCPA based on clinical, radiological, and microbiological evidence, and a total of 6 months of oral itraconazole $(400 \mathrm{mg} /$ day) was given to the test subjects. The overall response rate was measured on the basis of clinical response (improvement in cough, weight gain, and reduction in episodes of hemoptysis), radiological response (reduction in size/number of fungal ball, pulmonary infiltrates, and pleural fibrosis); and after 6 months, it was overall $76.5 \%$ in the itraconazole group as compared to control (35.7\%). They also reported adverse effects in $47 \%$ of the patients in the itraconazole group, but none of them were serious enough to warrant discontinuation of itraconazole. ${ }^{[17]}$

In study conducted by Kurhade et al., the prevalence and predisposing factors of Aspergillus infection in 123 patients were evaluated, out of which 20 patients were confirmed of having CPA. The authors used culture and serological studies to confirm the infection and also tested for the antifungal susceptibility of amphotericin-B, itraconazole, and fluconazole in terms of their minimum inhibitory concentration, and itraconazole was found to be most effective. The authors confirmed that combination of culture and serology was best for confirmation of diagnosis of CPA. ${ }^{[18]}$ In another prospective study by Shahid et al., they evaluated the prevalence of Aspergillus infection in 88 individuals with chronic lung disease. They conducted direct microscopy and fungal culture of BAL and antibodies against Aspergillus by immunodiffusion (ID) and enzyme-linked immunosorbent assay (ELISA). They also performed dot blot assay for anti-Aspergillus antibodies, which was confirmed by ID or ELISA. Aspergillus was isolated in culture from $13(14.7 \%)$ cases of chronic liver disease, while $30.6 \%$ cases showed anti-Aspergillus antibodies by serological methods. A. fumigatus was the predominant species isolated. 17 (19.3\%) cases of chronic lung disease showed antibody against Aspergillus by ID, 22 (25\%) by ELISA, while 19 of 27 seropositive cases also showed positive results by dot blot assay. They confirmed that ELISA was more sensitive tool than ID for the detection of anti-Aspergillus antibodies, and it is also useful in the detection of Aspergillus infection in culture-negative cases. ${ }^{[19]}$

The case series from India are summarized in Table 2.

\section{Declaration of patient consent}

The authors certify that they have obtained all appropriate patient consent forms. In the form the patient(s) has/have given his/her/their consent for his/her/their images and other clinical information to be reported in the journal. The patients understand that their names and initials will not be published and due efforts will be made to conceal their identity, but anonymity cannot be guaranteed.

\section{Financial support and sponsorship}

Nil.

\section{Conflicts of interest}

There are no conflicts of interest.

\section{RefERenCeS}

1. Denning DW. Invasive aspergillosis. Clin Infect Dis 1998;26:781-803.

2. Denning DW, Riniotis K, Dobrashian R, Sambatakou H. Chronic cavitary and fibrosing pulmonary and pleural aspergillosis: Case series, proposed nomenclature change, and review. Clin Infect Dis 2003;37 Suppl 3:S265-80.

3. Hope WW, Walsh TJ, Denning DW. The invasive and saprophytic syndromes due to Aspergillus spp. Med Mycol 2005;43 Suppl 1:S207-38.

4. Smith NL, Denning DW. Underlying conditions in chronic pulmonary aspergillosis including simple aspergilloma. Eur Respir J 2011;37:865-72.

5. Binder RE, Faling LJ, Pugatch RD, Mahasaen C, Snider GL. Chronic necrotizing pulmonary aspergillosis: A discrete clinical entity. Medicine (Baltimore) 1982;61:109-24.

6. Miller WT Jr., Sais GJ, Frank I, Gefter WB, Aronchick JM, Miller WT. Pulmonary aspergillosis in patients with AIDS. Clinical and radiographic correlations. Chest 1994;105:37-44.

7. Pasqualotto AC, Denning DW. An aspergilloma caused by Aspergillus flavus. Med Mycol 2008;46:275-8.

8. Severo LC, Geyer GR, Porto Nda S, Wagner MB, Londero AT. Pulmonary Aspergillus Niger intracavitary colonization. Report of 23 cases and a review of the literature. Rev Iberoam Micol 1997;14:104-10.

9. Denning DW. Chronic forms of pulmonary aspergillosis. Clin Microbiol Infect 2001;7:25-31.

10. Denning DW, Cadranel J, Beigelman-Aubry C, Ader F, Chakrabarti A, Blot $\mathrm{S}$, et al. Chronic pulmonary aspergillosis: Rationale and clinical guidelines for diagnosis and management. Eur Respir J 2016;47:45-68. 
Marwah, et al.: Chronic pulmonary aspergillosis - Case series

11. Kosmidis C, Denning DW. The clinical spectrum of pulmonary aspergillosis. Thorax 2015;70:270-7.

12. Pai Jakribettu R, George T, Abraham S, Fazal F, Kinila S, Baliga MS. Clinical and laboratory profile of chronic pulmonary aspergillosis: A retrospective study. Egypt J Bronchol 2019;13:109-13.

13. Takazono T, Izumikawa $\mathrm{K}$. Recent advances in diagnosing chronic pulmonary aspergillosis. Front Microbiol 2018;9:1810.

14. Sehgal I, Choudhary H, Dhooria S, Agarwal A, Garg M, Chakrabarti A, et al. Is there an overlap in immune response between allergic bronchopulmonary and chronic pulmonary aspergillosis? J Allergy Clin Immunol 2018:7:969-74.

15. Sehgal IS, Choudhary H, Dhooria S, Aggarwal AN, Garg $M$, Chakrabarti A, et al. Diagnostic cut-off of Aspergillus fumigatus-specific IgG in the diagnosis of chronic pulmonary aspergillosis. Mycoses
2018;61:770-6.

16. Chawla K, Kosaraju K, Rayasam S, Mukhopadhyay C. Clinico-microbiological profile of chronic pulmonary aspergillosis from a tertiary care centre in southern India. J Clin Diagn Res 2013;7:2712-5.

17. Agarwal R, Vishwanath G, Aggarwal AN, Garg M, Gupta D, Chakrabarti A. Itraconazole in chronic cavitary pulmonary aspergillosis: A randomised controlled trial and systematic review of literature. Mycoses 2013;56:559-70.

18. Kurhade AM, Deshmukh JM, Fule RP, Chande C, Akulwar S. Mycological and serological study of pulmonary aspergillosis in central India. Indian J Med Microbiol 2002;20:141-4.

19. Shahid M, Malik A, Bhargava R. Prevalence of aspergillosis in chronic lung diseases. Indian J Med Microbiol 2001;19:201-5. 